Table 6. The Training of Astronomy Club Leaders at the

Eötvös University of Budapest (Compulsory Courses)

\begin{tabular}{|c|c|c|c|c|c|c|}
\hline Semesters: & $1 \mathrm{st}$ & 2nd & 3 th & 4 th & 5 th & 6 th \\
\hline General Astronomy & + & + & + & + & - & - \\
\hline General Astrophysics & + & + & + & + & - & - \\
\hline History of Astronomy & + & + & + & + & + & + \\
\hline Celestial Mechanics & - & - & + & + & - & - \\
\hline Mathematics & + & + & - & - & - & - \\
\hline Physics & + & + & - & - & - & - \\
\hline Computers in Astronomy & - & - & + & + & - & - \\
\hline Astronomical Telescopes & - & - & + & + & - & - \\
\hline Astronomical Photography & - & - & - & - & + & + \\
\hline Astronomical Measurements & - & - & - & + & + & - \\
\hline Methods of Popularization of Astronomy & - & - & - & - & + & + \\
\hline The Use of Visual Aids & - & - & - & - & - & + \\
\hline
\end{tabular}

\title{
GRADUATE ASTRONOMY STUDIES AT THE UNIVERSITY OF SÃO PAULO, BRAZIL
}

\section{Walter J. Maciel}

Departamento de Astronomia, Instituto Astronômico e Geofisico da USP, Caixa Postal 30.627, 01051 São Paulo SP, Brazil

\section{Introduction}

The University of São Paulo (USP), founded in 1934, is the largest university in Brazil, having about 50,000 students and 5,000 teachers/researchers distributed among 33 institutes and 184 departments.

Astronomical work was already developed at the older São Paulo Observatory (founded 1912), which was later attached to the university. The observatory has been renamed as Instituto Astronomico e Geofisico (IAG), and presently houses the departments of Astronomy, Geophysics, and Meteorology. Graduate astronomy courses, intended to provide Master (M.Sc.) and Doctor (D.Sc.) degrees, started in 1973.

\section{Astronomical Research at the USP}

The astronomical research at the USP is basically conducted by the IAG. The main fields of research include theoretical and observational projects in the following areas: Fundamental Astronomy, Mathematical and Dynamical Astronomy, and As- 
trophysics (solar system, stars, the interstellar medium, galaxies, and astrophysical plasmas). The main instruments include a meridian circle, a Danjon astrolabe, and a $60-\mathrm{cm}$ Boller and Chivens reflector, located at the nearby "Abrahão de Moraes Observatory." Instruments of the National Astrophysical Laboratory (LNA), which include a $1.6-\mathrm{m}$ Boller and Chivens reflector, are normally used by the staff, and several projects are conducted at the ESO and U.S. observatories in Chile. The present staff consists of 32 people, 17 of whom have a Ph.D. or equivalent degree; the remaining are masters still working on their doctoral theses.

\section{Astronomy Courses}

The university does not provide a college degree in astronomy, and candidates for graduate studies are strongly recommended to complete a 4-year Physics program. Several one-semester undergraduate courses in astronomy are offered to students majoring in Physics, thus forming an introductory basis to further graduate work.

The candidate for the M.Sc. degree must take a series of one-semester courses from a minimum of six. Additional short courses by visiting scientists are often scheduled. The average student takes about two years to conclude the courses, and then takes a qualifying examination. At the same time, he or she engages in a research project for the M.Sc. dissertation, which is finished on average after 4 years of graduate work. The D.Sc. candidate takes 3 more courses plus a new qualifying examination. After a minimum of 3 more years, he or she will have produced a thesis based on an original investigation, which will be published in paper form in international astronomical journals.

\section{Discussion}

Through the end of 1987 , the total number of students enrolled in the M.Sc. and D.Sc. programs were 138 and 65 , respectively; 58 of them have obtained the M.Sc. degree, and 14 obtained the D.Sc. degree. The present number of students (December 1987) is 26 (M.Sc. course) and 41 (D.Sc. course).

After an initial period when most students were enrolled in the M.Sc. program, in recent years this tendency has been inverted. There were 138 candidates for the M.Sc. degree in the 15-year period, out of which $54(39 \%)$ left the program and 58 $(42 \%)$ concluded it. The attrition rate is much lower among candidates for the D.Sc. degree, where out of 65 candidates, $10(15 \%)$ have left the program, and $14(22 \%)$ concluded it. However, several students who left the program have in fact enrolled in another one in the country, so that the trial period effectively occurs during the M.Sc. program. All candidates for the D.Sc. degree who concluded the program have obtained jobs in the country. Among the masters, $27(59 \%)$ have also obtained jobs, which high-percentage reflects the lack of qualified astronomers in the country.

(Work partly supported by FAPESP and CNPq - Brazil.) 